\title{
English-Arabic Hybrid Machine Translation System using EBMT and Translation Memory
}

\author{
Rana Ehab ${ }^{1}$, Mahmoud Gadallah ${ }^{3}$ \\ Computer Science Department, Modern Academy for \\ Computer Science and Management Technology \\ Cairo, Egypt
}

\author{
Eslam Amer ${ }^{2}$ \\ Computer Science Department \\ Misr International University \\ Cairo, Egypt
}

\begin{abstract}
The availability of a machine translation to translate from English-to-Arabic with high accuracy is not available because of the difficult morphology of the Arabic Language. A hybrid machine translation system between Example Based machine translation technique and Translation memory was introduced in this paper. Two datasets have been used in the experiments that were constructed by using internal medicine publications and Worldwide Arabic Medical Translation Guide Common Medical Terms sorted by Arabic. To examine the accuracy of the system constructed four experiments were made using Example Based Machine Translation system in the first, Google Translate in the second and Example Based with Google translate in the third and the fourth is the system proposed using Example Based with Translation memory. The system constructed achieved 77.17 score for the first dataset and 63.85 score for the second which were the highest score using BLEU score.
\end{abstract}

Keywords-Hybrid machine translation system; translation memory; internal medicine publications; google translate; $B L E U$

\section{INTRODUCTION}

In 1952 the first conference on MT came. There was the first demonstration of a translation system in January 1954, and it attracted a great deal of attention and since then there has been no stopping [1]. Since Language technologies are very successful nowadays Machine Translation has been applied to the medical domain [2]. The quality of language technologies is growing very rapidly [2]. People with different languages can share ideas and information worldwide on every topic as business, economic, educational, political, socio-cultural, etc. if machine translation researchers have the ability to develop a perfect multilingual machine translation system [3]. The presence of a machine translation that has the ability to translate any text in any domain at the required quality is expected in not-too-distant future [2]. Machine translation must present a reasonable approach to translate terms to meet commercial needs [4]. Generally users are interested in obtaining a rough idea of a text's subjects or what it means [2]. However, some applications require much more than this [2]. As example, in the medical field the beauty and correctness of the text may not be important, but the precision and efficiency of the translated message are very important [2]. Machine translation systems can be used to translate medical records [2].

The most important task for saving with high-quality medical services is the communication between medical physicians and patients [5]. If medical physicians and patients do not share a common language, the diagnosis and treatment will be more difficult due to the language barrier that prevents effective communication [5]. Another case is people who travel to receive high-quality or affordable medical treatment that is not available in their home country [5]. When translating medical information and make it understandable both physicians and patients will benefit [6]. As an example, Healthcare Technologies for the World Traveller confirm that a foreign patient may need a description of their diagnosis with a related and full set of information [2].

The world has become a small village because of the rapid changes in information and communication technology via internet where people from all over the world can connect with each other in dialogue and communication [7]. The translation databases and translator workstation such as the Google Translate (GT), Bing Translate, Yahoo, Babel Fish and Systran that were developed and influenced by the internet was the development of computer-based translation tools [8].

Using websites in translation has been outspread. However, the task of translating a medical text is not as easy as translating any other English text because of the complex information that it contains. So, using existed systems in translation a medical text produces a translation text with some problems. Because of the difference in language categories, current methods are far from being at the degree where they can be of practical use especially in English-toArabic medical translation.

In the medical domain most institutional and research information is available as English text [9], if people don't know English language well they will not be able to make use of these information without a help [10]. So, the task is helping everyone to use web and this will be achieved by automatic language translators [10]. Because of the flow of information in foreign languages through web the use of machine translation technology is must [11].

Most of the researches in Arabic Machine Translation are mainly concentrated on the translation between English and Arabic because English is a universal language [12]. This will help in simplifying the Arab communication with other countries [12]. That was the reason to choose translating from English to Arabic. 
The field of Machine Translation research is largely controlled by corpus-based nowadays, or data- driven approach [13]. Although Example Based Machine Translation (EBMT) and Statistical Machine Translation (SMT) are from corpus-based model each of them has their own advantages and disadvantages [14]. Example Based Machine Translation can works well with a limited training and testing datasets other than Statistical Machine Translation that needs a large dataset to result a significant translation [15]. Also when the nature of the training and test are close the Example Based Machine Translation System works well. Also reusing the segment of a test sentence that can be found in the source side of the example-base improves the translation by Example Based Machine Translation Systems. The idea of Example Based Machine Translation is getting translation examples of similar sentences.

Using Example Based Machine translation is often linked with using another technique called "Translation Memory" [15]. Translation memory (TM), is a database that is today widely used Computer-Assisted Translation (CAT) tool prepared for future reuse of already translated texts [16]. The similarity between them is they both reuse the examples from the existing translations. The main difference between them is that Example Based Machine Translation is an automated technique for translation whereas Translation Memory is an interactive tool for the human translator [15].

Beside the technique used to build the translation system the dataset that is used in training and testing of the system is important. Some machine translation systems evaluation was low because of the dataset used. So, when building a machine translation system it is very important to consider the goodness of the dataset that will be used. So, in the experiments in this paper two datasets were used. The first dataset constructed using internal medicine publications from [17]. The second one constructed using internal medicine publications and Worldwide Arabic Medical Translation Guide Common Medical Terms sorted by Arabic which is an English-Arabic medical dictionary that will be described later.

The attempt to use Example based machine technique and Translation memory to translate English medical text to Arabic medical text will be described in this paper. As the constructed datasets were not large the choice to use Example based machine technique that works well with a limited training and testing datasets was the best. Also with the advantages of Translation memory which are consistency, speed and cost-saving [16] that will benefit the resulted translation. Also, from the benefits of using Translation memory: need for consistent use of terminology, data sharing of common resources, re-use of already translated and revised text suggest used of Translation Memory, in its simplest form a database [16].

The rest of the paper is organized as following : the second section describes the recently related works in machine translation in medical domain and non-medical domain, the third section describes the issues that face medical domain, the forth section describes the datasets and the hybrid translation system from English to Arabic that was built, the fifth section describes the experiments to evaluate the system built, the sixth section shows the evaluation of the experiments using BLEU metric and finally the last section shows a brief conclusion of the work.

\section{RELATED WORK}

In the medical domain there are many machine translation systems for various languages have been developed using different approaches of machine translation. Also machine translation has been developed to translate English text to Arabic text but not in medical domain.

Dandapat, et al. [15] used Example-based machine translation and Translation Memory to translate medical text from English to Bangle. They translated receptionist dialogues of medical and primarily appointment scheduling. Their first step was to collect their data and then building a Translation Memory automatically from a corpus of patient dialogue using Moses toolkit. They created two Translation Memories the first contains phrase pairs that are aligned and the second one contains the word aligned file [15].

They made five different experiments to show the accuracy of their system. The fourth experiment achieved the highest accuracy which is 57.56 [15] where they used their system with the first and second Translation Memories. However they achieved the highest accuracy, some errors appeared, the first was the wrong of source-target equivalent in both Translation Memory systems [15]. The second in the recombination step that some words are translated separately [15].

Névéol, et al. $\lceil 18\rceil$ built a statistical machine translation system to translate systematic reviews from English to French. They used three different datasets. They made five systems. During the evaluation the last system achieved the best accuracy which was (40.00 BLEU) [18] where they used Cochrane translation table and an integrated translation table between EMEA and WMT. Also, Subalalitha, et al. [19] tried to use statistical machine translation to translate from English to Hindi and achieved accuracy (73.43).

Renato, et al. [20] discussed translating clinical term descriptions from Spanish to Brazilian Portuguese. HIBA dictionary was used as a Spanish dictionary. They collected medical terms of Portuguese language using several sources. They made two experiments and evaluated them. For both experiments they used for translation Bing, Google Translate and their system M-SMT. In the first experiment their system achieved the highest score which is (58.9) [20] using BLEU score. In the second experiment their system achieved (86.7) [20]. That shows that their system achieved the highest score.

As showed that the second experiment achieved higher scores in all translation systems. However although they achieved high scores there were some errors as [20]: OOV words are usually translated into English or left in Spanish, a part of the corpus had words with spelling in European Portuguese, Compound medical terms, especially drugs with a hyphen, possibly misaligned in training.

$\mathrm{Li}$, et al. [21] developed a hybrid translation system between Dictionary based machine translation technique and Statistical machine translation technique. They translated 
query terms in medical domain from English to German and vice versa [21]. Their corpus was a mix from more than one corpus. They made two experiments to evaluate their system. In the first one they used Phrase-based machine translation system and in the second one they used their system [21]. According to their evaluation they achieved better evaluation than the first one. Their system achieved (15.3) for translating from English to German and (24.5) from German to English which was higher than the accuracy of the other system.

Wołk, et al. [22] goal was to translate medical data from English to Polish and vice versa so they developed a SMT system for this purpose. For their dataset they used the European Medical Agency (EMEA) data. To evaluate their system they made 13 experiments. The results showed that translating from Polish to English evaluates better than translating from English to Polish [22] The fifth experiment achieved the highest score among the other experiments that was (76.34) for BLEU score for translation from Polish to English and (73.32) for translating from English to Polish [22]. Also Johanna Johnsi Rani G, et al. [23] used SMT to translate medical reports from English to Tamil. They evaluated their system with the results of Google Translate and they achieved better accuracy.

Wołk, et al. [2] built a machine translation system rely on using neural network. They used European Medicines Agency (EMEA) parallel corpus to derive their corpus. The system translates Polish medical text into English medical text and vice versa [2]. They made three experiments to evaluate their system. Their system achieved (24.32) for translating from Polish-to-English and (17.50) for translating from English-toPolish [2] that was lower than the other two experiments. Also Artetxe, et al. [24] tried to use neural network to translate from French and German to English but they achieved low accuracy.

Amer, et al. [25] built a query translation system which is Wiki transpose for cross-lingual information retrieval (CLIR) that relied on Wikipedia as a source for translations. They used the system to check how reliable Wikipedia is to get corresponding translation coverage of English to Portuguese and also Portuguese to English queries [25]. For their evaluation they made two experiments. They used English Open Access, Collaborative Consumer Health Vocabulary Initiative dataset in the first experiment [25]. They used a collection of Portuguese medical terms that were rated by medical experts as medical terms in the second experiment. A coverage ratio in Wikipedia about $81 \%$ and about $80 \%$ [25] in single English and Portuguese terms respectively was reached.

Rana Ehab, et al. [17] built a machine translation system using Example based machine translation technique to translate English medical sentences to Arabic medical sentences. They constructed their parallel corpus using the internal medicine publications for internal diseases only [17]. The matching stage was used from Example based technique to find the closest example from the parallel corpus as the example based for the system. The second experiment made using Google translate and the same data were translated to examine the accuracy of their system but Google translate achieved higher score than their system. Google translate achieved (53.56) for BLEU score and their system achieved (48.86) [17].

Shaalan, et al. [12] built a translation system to translate English noun phrase into Arabic. They used Transfer machine translation approach as their system [12]. They evaluated their system by using 50 titles from the computer science domain as training dataset for their system and for testing they used other 66 new real thesis titles from the computer science domain. Their evaluation showed that the system translated 47 noun phrases correctly and the remaining 109 noun phrases have problems [12].

Shaalan, et al. [26] built a translation system using Rulebased transfer machine translation technique to translate expert systems in the agriculture domain from English to Arabic and vice versa. This translation process includes translating knowledge base, in particular, prompts, responses, explanation text, and advices. Those expert systems are built in CLAES $^{1}$ [26].

They used for their system a set of real parallel 100 phrases and sentences from both English and Arabic versions of agricultural expert systems at CLAES that were used as a gold standard reference test data [26]. They made the evaluation through two experiments. The second experiment achieved higher accuracy than the first which is 0.6427 for English to Arabic direction and 0.8122 for Arabic to English direction [26]. Also Kouremenos, et al. [27] used also Rulebased technique to translate Greek to Greek Sign language.

Al-Taani, et al. [28] translated well-structured English sentences into well-structured Arabic sentences using rule based approach. They used 184 English proverbs from AlMawrid, English- Arabic dictionary [28]. Also they used 125 well structures English sentences from many text books. During the evaluation $57,3 \%$ of the first dataset translated correctly and 84.6 of the second dataset translated correctly [28]. These results were not as they supposed because of many reasons. From these reasons that proverbs have no specific structure, also proverbs are much related to the culture of some nations [28]. Also Mouiad Alawneh, et al. [3] translated well-structured English sentences into well-structured Arabic sentences but using Grammar parser and example based machine translation technique.

As shown in the previous approaches of machine translation in medical domain most of them used Statistical machine translation technique and Example based machine translation technique. There was also an attempt to use neural network in translation but in comparison with SMT the second achieved higher score. Also an approach [15] used Translation memory with Example based technique and achieved higher scores than using Example based technique with SMT. For this reason the proposed system is to build a system using Example based machine translation and Translation memory. Also as shown that most of English to Arabic machine translation systems in non-medical domain used Rule based machine translation approach as they need to analyze the English text in terms of morphology, syntax and semantic

\footnotetext{
${ }^{1}$ Stands for Central Laboratory of Agricultural Expert Systems (CLAES), Agricultural Research Centre (ARC), Egypt, http://www.claes.sci.eg
} 
which is not important for English text in medical domain. The strengths of rationalism method and empiricist method are merged through using Hybrid machine translation [29].

\section{ISSUES WITH MEDICAL DOMAIN}

In [17] to construct an efficient Machine Translation system for a Medical Domain there are two main issues which are: parallel corpus collection, size and type of corpus. Beside them there is a third issue which is building a Translation Memory [15]. The medical terms are different from any other English terms. For that building an efficient medical corpus is not an easy task. To evaluate the system two datasets were used. The first one is [17] where they used internal medicine publications to build it. The second dataset constructed using internal medicine publications and Worldwide Arabic Medical Translation Guide Common Medical Terms sorted by Arabic which is an English-Arabic medical dictionary to build English-Arabic parallel corpus. The first corpus consists of 259 medical sentences; for each sentence there are 8 words on average [17]. The second corpus consists of 509 medical sentences.

The proposed system uses Example-Based Machine Translation which is a data-driven machine translation technique $[15,17]$ that needs a machine readable parallel corpus. So when building such a system how many examples needed musy be known? In a comparison with such systems the first corpus is very small but the second corpus is larger than other corpuses as in Tabel 1. The first corpus is small because it is built from only the medical data of internal diseases but the second corpus includes more diseases besides using Worldwide Arabic Medical Translation Guide Common Medical Terms sorted by Arabic. As seen in Table 1. many systems have been constructed using a small corpus.

As there is no access to an existed Translation Memory building a Translation Memory automatically for the proposed system using ${ }^{2}$ Moses toolkit was considered. A Translation memory was created based on word aligned file created using Moses word alignment (Giza++) [15]. Because each source word has multiple target equivalents all the multiple equivalent words in sorted order were kept. This Translation memory will help in the second stage of the system which is finding the alignment between the result from the database and its translation.

TABLE I. Source of Medical Terms of Portuguese Language

\begin{tabular}{|l|l|l|}
\hline System & Language Pair & Size \\
\hline TTL & English-> Turkish & 488 \\
\hline TDMT & English-> Japanese & 350 \\
\hline EDGAR & German-> English & 303 \\
\hline ReVerb & English-> German & 214 \\
\hline ReVerb & Irish -> English & 120 \\
\hline METLA-1 & English -> French & 29 \\
\hline METLA & English -> Urdu & 7 \\
\hline
\end{tabular}

\footnotetext{
${ }^{2}$ Moses (http://www.statmt.org/moses/) is a SMT system that automatically trains a translation model for any language pair.
}

\section{OUR APPROACH}

\section{A. Data Preparation}

In each domain words have different meanings so, their translation has to fit in the excepted representation in the domain. Therefore to ensure that they are treated consistently throughout the technical text, it is important to identify them correctly [30].

In the previous section, as mentioned two datasets were used. The first one was constructed by [17] where they built it from the indications and side effects from the internal medicine publications in both languages English and Arabic for internal diseases only.

The second dataset were constructed from indications and side effects from the internal medicine publications for multiple diseases and Worldwide Arabic Medical Translation Guide Common Medical Terms sorted by Arabic .After that, some processing on English data were made as tokenization, a lower casing, and final cleaning. Pre-processing Arabic sentences could change the meaning of the sentence due to the morphology of the language and the meaning of the sentence is very sensitive in the medical domain .So, no pre-processing for the Arabic will be done.

\section{B. Translation System}

In the example based translation, a system is defined which contains a set of source language sentences and corresponding target language sentences. During the run time, example based translation use bilingual corpus as its database. This database is stored in the translation memory. In translation memory, the user translates text these translations are added to a database, and when the same sentence occurs again during the translation, the previous translation is inserted in to the translated document. The advantage of the example based translation the translation memory saves the user effort of re translating the sentence and this saves the processor time and also the user time. EBMT can help to overcome some of the weaknesses of the other approaches [31].

With the advantages of the Example Based Machine Translation approach and the Translation Memory a hybrid system that uses both of them to translate English medical sentence to Arabic medical sentence was developed. Arabic language was chosen as destination language because there is many possible ways to express the same sentence in Arabic that provides a significant challenge to MT [3]. The accents of modern Arabic are well-known as having agreement asymmetries that are sensitive to word order effects. As all Example Based Machine Translation system the proposed approach is from three stages which are: Matching, Adaption and Recombination [15].

1) The proposed hybrid machine translation system: The hybrid machine translation system in Fig. 1 is used to translate medical sentences from English to Arabic using Example Based machine translation and Translation memory.

User initially inserts the input English sentence, the sentence then goes to some pre-processing steps: tokenization, lower casing and stop word removing, then the sentence sent 
to Example based which is the parallel corpus to find the closest example by computing edit distance between the input sentence and each example and this will be discussed later. The example that gets the highest score will be the closest example. Then using the parallel corpus the translation of the closest example will be gotten.

Then the alignment between the input sentence and the example will be found to find the unmatched portions, this done while computing the edit distance. Also the alignment between the example and its translation example will be found to find the unmatched portions by using the Translation memory and this will be described in section 4.2.3. Then the unmatched portions of the input sentence will be replaced with the unmatched portions of the translated sentence and add or substitute from the translated sentence and this will be discussed later.

Finally the un-translated segments that were replaced will be translated and added to the translated sentence using the translation memory and then the final translated sentence was get.

2) Matching stage: In this stage the task is to find the source closest examples from the database that closely matches the input sentence and that is done by using wordbased edit distance metric (1) (Levenshtein, 1965; Wagner and Fischer, 1974) [16].

Score $(\mathrm{Si}, \mathrm{Se})=1-\frac{\mathrm{ED}(\mathrm{Si}, \mathrm{Se})}{\max (|\mathrm{Si}|,|\mathrm{Se}|)}$

Where Si denotes the input sentence and Se denotes the example from the database sentence. So, $|S i|$ and $|\mathrm{Se}|$ denotes the length of an input sentence and example sentence extracted from database and $\operatorname{ED}(\mathrm{Si}, \mathrm{Se})$ refers to the word based edit distance between $S i$ and $S e$.

Based on the above scoring technique the following examples from the database in (2) for the input sentences in (1) were gotten.

(1) a- impaired function of the liver

b- arthrosis

c- nasal congestion

(2) a- impaired function of the kidneys

b- arthritis

c- lung congestion

Then the associated translation St in (3) was gotten for the sentences in (2) from the database. This translation will be used in the following subsections to get new translation texts.

خلل لوظائف الكلى- a (3)

b- التهاب

c- احتقان الرئنة

3) Adaption stage: In this stage the unsuitable fragments from the resulted translation from the previous stage were extracted. For this purpose the three sentences that have gotten from the previous stage will be aligned, which were: input sentence $\mathrm{Si}$, the closest example of the source $\mathrm{Se}$ and its translation St.

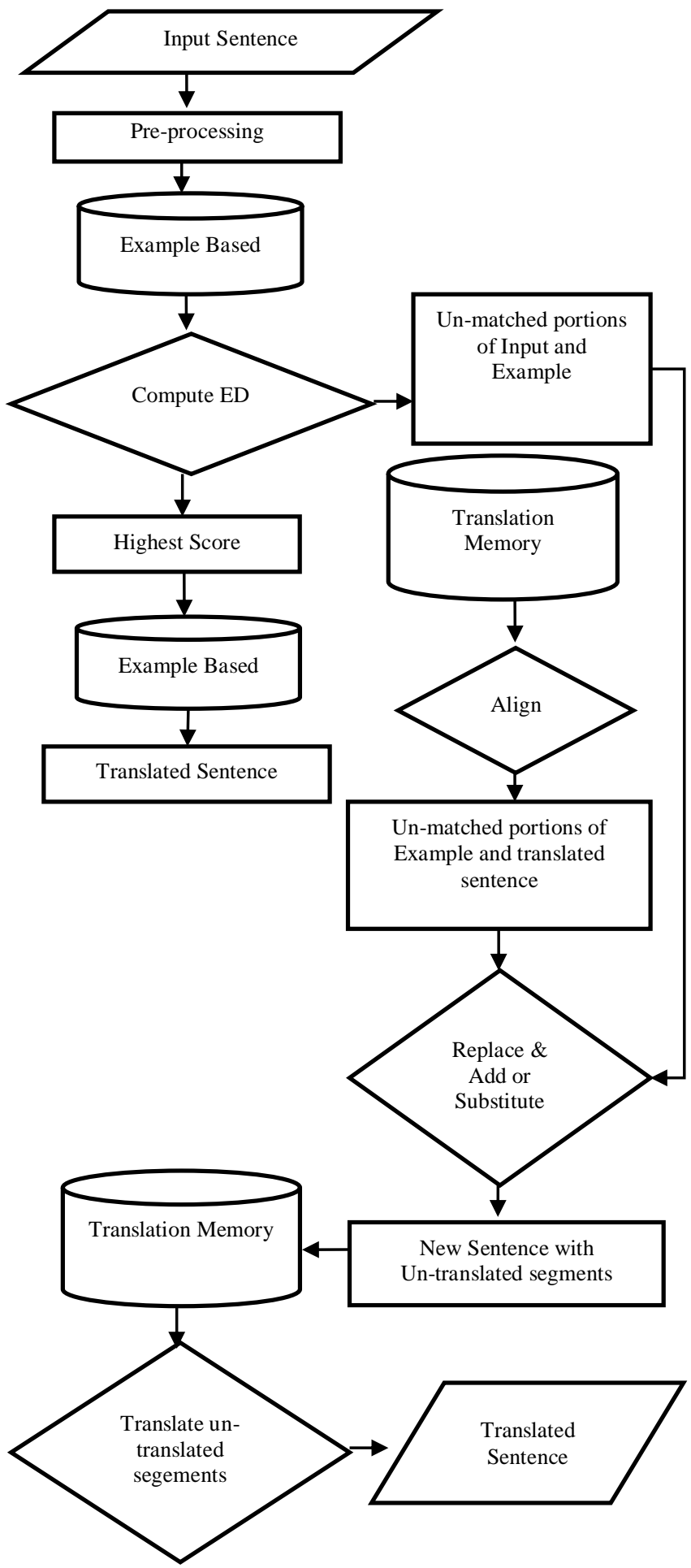

Fig. 1. Hybrid Machine Translation System.

Aligning the input sentence $\mathrm{Si}$ and the closest example $\mathrm{Se}$ is done while computing the edit distance in equation (1). This is shown in example (4) (4a1) with (4a2) are aligned, in (5) (5a1) with (5a2) are aligned and in (6) (6a1) with (6a2) are aligned. Then the closest example Se with its translation $\mathrm{St}$ will be aligned by using the Translation memory that was built and as shown (4a2) with (4a3) are aligned, (5a2) aligned with 
(5a3) and (6a2) aligned with (6a3). In the next stage the unmatched fragments will be replaced and the matched fragments will keep unchanged.

(4) a-1-impaired function of the [1:liver ]

2- impaired function of the [1: kidneys]

3"

(5) a- 1- [1:arthrosis]

2- [1:arthritis]

3- [1: التهاب]

(6) b-1-[1: nasal] congestion

2- [1:lung ] congestion

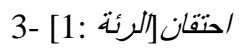

Recombination stage: After extracting the unsuitable fragments in the previous stage the next purpose is to adjust the resulted translation. This is done by adding or substituting the fragments from the input sentence $(\mathrm{Si})$ with the translation equivalent sentence (St) [16]. From example (4) \{الكلى الكلى $\}$ need to be replaced from (4a3) with \{liver\} from (4a1), from example (5) \{ / لتهاب $\}$ need to be replaced from (5a3) with \{ arthrosis $\}$ from (5a1) and from example (6) \{ لرئة replaced from (6a3) with $\{$ nasal $\}$ from (6a1). And the results will be the sentence in (7), (8) and (9).

خلل لوظائف (7) liver

(8) arthrosis

(9) nasal (حتقان

During the aligning the alignment might not only one to one align. If the input sentence ( $\mathrm{Si}$ ) has extra segments that have no align to translation equivalent sentence $(\mathrm{St})$ this segments are added to the final resulted sentence but if there is extra segments in the translation equivalent sentence $(\mathrm{St})$ they will be deleted from the final resulted sentence. After this step the task is to translate the un-translated segments using two methods. The first method is to use the translation memory to get the translation of the un-translated segments. The second method is to use Google translate as a statistical machine translation to get the translation of the un-translated segments. The final result of the translation using Translation memory showed in (10), (11) and (12).

$$
\begin{aligned}
& \text { خلل لوظائف الكبد (10) } \\
& \text { تييس (11) } \\
& \text { احنقان الأنف (12) }
\end{aligned}
$$

\section{RESULTS AND DISCUSSION}

As said before two datasets were used in the experiments for each dataset four experiments were made to measure the accuracy of the proposed system using bilingual evaluation understudy (BLEU) matrix. The datasets were divided to one word sentences, two word sentences and multiple word sentences and for each the experiments were made. In the first experiment Google translate was used as it is a statistical machine translation [32] that is widely known with its robustness, good performance, and the fact that it does not require manually crafted rules [33] to translate the input sentences. In the second experiment EBMT was used from it matching stage only was used and the closet translation was gotten and takes it as the translation for the input sentence. In the third experiment the translation memory was used in recombination stage to translate the unmatched portions. In the fourth experiment Google Translate was used in recombination stage to translate the unmatched portions. BLEU score was used to automatically evaluate the proposed system. BLEU score captures the fluency of the translation.

The following tables (Table 2 and Table 3) where the four experiments were made for the whole dataset shows the accuracy over the two datasets and as shown when using the proposed system that uses both Example Based Machine Translation and Translation Memory the results where the best over the other techniques.

Results in Table 4 and Fig. 2 also in Table 5 and Fig. 3 show that over one word translation, two words translation and multi-words translation the proposed approach achieved the highest score over the four experiments and using Google translate to translate the un-matched portions shows a very bad score. Also as shown when the input sentence is from multi-words the score increased.

As shown in Table 6 most of machine translation systems in medical domain used Statistical machine translation technique that will cause little accuracy with the dataset used because of the Arabic morphology and the size of the corpus. Their datasets were from systematic, clinical descriptions, queries where they are from hospitals data but the core of the used dataset were from internal medicine publications that are used daily by patients and may contain complex data that need translation.

TABLE II. SYSTEMS ACCURACIES FOR THE FIRST DATASET

\begin{tabular}{|l|l|}
\hline System & BLEU \\
\hline Google Translate & 53.56 \\
\hline EBMT & 48.86 \\
\hline EBMT+ Translation Memory & 77.17 \\
\hline EBMT+ Google & 73.07 \\
\hline
\end{tabular}

TABLE III. SYSTEMS ACCURACIES FOR THE SECOND DATASET

\begin{tabular}{|l|l|}
\hline System & BLEU \\
\hline Google Translate & 51.06 \\
\hline EBMT & 50.82 \\
\hline EBMT+ Translation Memory & 63.85 \\
\hline EBMT+ Google & 61.43 \\
\hline
\end{tabular}

TABLE IV. SYSTEMS ACCURACIES FOR THE FIRST DATASET FOR DIFFERENT INOUTE SIZE

\begin{tabular}{|l|l|l|l|l|}
\hline $\begin{array}{l}\text { System } \\
\text { Accuracy }\end{array}$ & $\begin{array}{l}\text { Google } \\
\text { Translate }\end{array}$ & EBMT & $\begin{array}{l}\text { EBMT+ } \\
\text { Translation } \\
\text { Memory }\end{array}$ & $\begin{array}{l}\text { EBMT } \\
+ \\
\text { Google }\end{array}$ \\
\hline $\begin{array}{l}1 \text { word } \\
\text { translation }\end{array}$ & 51.42 & 41.52 & 66.02 & 48.41 \\
\hline $\begin{array}{l}2 \text { words } \\
\text { translation }\end{array}$ & 51.32 & 47.36 & 59.21 & 19.89 \\
\hline $\begin{array}{l}\text { Multi words } \\
\text { translation }\end{array}$ & 54.23 & 52.99 & 80.93 & 74.47 \\
\hline
\end{tabular}




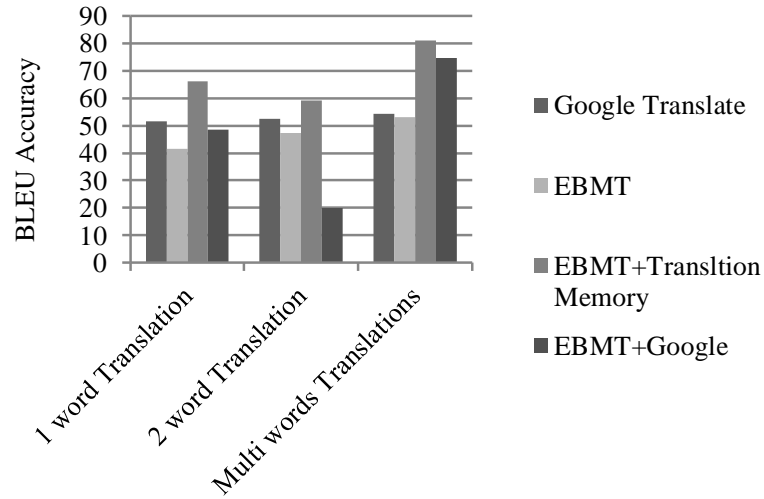

Fig. 2. Comparison between Systems Accuracies for the First Dataset for Different Input Size in the First Dataset.
TABLE V. SYSTEMS ACCURACIES FOR THE SECOND DATASET FOR DIFFERENT INOUTE SIZE

\begin{tabular}{|c|c|c|c|c|}
\hline $\begin{array}{c}\text { System } \\
\text { Accuracy }\end{array}$ & $\begin{array}{c}\text { Google } \\
\text { Translate }\end{array}$ & EBMT & $\begin{array}{c}\text { EBMT+ } \\
\text { Translation } \\
\text { Memory }\end{array}$ & $\begin{array}{c}\text { EBMT } \\
+ \\
\text { Google }\end{array}$ \\
\hline $\begin{array}{c}1 \text { word } \\
\text { translation }\end{array}$ & 35.33 & 50.82 & 30.20 & 14.31 \\
\hline $\begin{array}{c}2 \text { words } \\
\text { translation }\end{array}$ & 44.71 & 50.70 & 28.46 & 16.79 \\
\hline $\begin{array}{c}\text { Multi words } \\
\text { translation }\end{array}$ & 54.09 & 52.99 & 72.49 & 53.56 \\
\hline
\end{tabular}

TABLE VI. COMPARISON WITH OTHER SYSTEMS

\begin{tabular}{|c|c|c|c|}
\hline Reference number & Technique & Dataset type & The proposed system \\
\hline 18 & statistical machine translation system & $\begin{array}{l}609 \text { systematic reviews from English } \\
\text { to French }\end{array}$ & \multirow{9}{*}{$\begin{array}{l}\text { EBMT+ Translation memory } \\
\text { (translation system) } \\
\text { And the dataset used is using interna } \\
\text { medicine publications and Worldwide } \\
\text { Arabic Medical Translation Guide } \\
\text { Common Medical Terms sorted by } \\
\text { Arabic which is an English-Arabic } \\
\text { medical dictionary } \\
\text { Translating from English to Arabic }\end{array}$} \\
\hline 19 & statistical machine translation system & English to Hindi & \\
\hline 20 & statistical machine translation system & $\begin{array}{l}\text { clinical term descriptions from } \\
\text { Spanish to Brazilian Portuguese }\end{array}$ & \\
\hline 21 & $\begin{array}{l}\text { Dictionary based machine translation } \\
\text { technique and Statistical machine } \\
\text { translation technique }\end{array}$ & $\begin{array}{l}\text { query terms in medical domain from } \\
\text { English to German and vice versa }\end{array}$ & \\
\hline 22 & statistical machine translation system & $\begin{array}{l}\text { medical data from English to Polish } \\
\text { and vice versa }\end{array}$ & \\
\hline 23 & statistical machine translation system & $\begin{array}{l}\text { medical reports from English to } \\
\text { Tamil }\end{array}$ & \\
\hline 2 & Neural networks & $\begin{array}{l}\text { medical data from English to Polish } \\
\text { and vice versa }\end{array}$ & \\
\hline 24 & Neural networks & French and German to English & \\
\hline 17 & $\begin{array}{l}\text { Example based machine translation } \\
\text { technique matching stage }\end{array}$ & $\begin{array}{l}\text { the internal medicine publications for } \\
\text { internal diseases }\end{array}$ & \\
\hline 12 & Transfer Approach & $\begin{array}{l}50 \text { titles from the computer science } \\
\text { domain for training } \\
66 \text { real thesis titles from the computer } \\
\text { science domain for testing }\end{array}$ & \multirow{4}{*}{$\begin{array}{l}\text { Dataset is Medical Text. } \\
\text { Using Eaxmple Based technique with } \\
\text { Translation Memory }\end{array}$} \\
\hline 26 & $\begin{array}{l}\text { Rule-based transfer machine } \\
\text { translation technique }\end{array}$ & $\begin{array}{l}100 \text { phrases and sentences from both } \\
\text { English and Arabic versions of } \\
\text { agricultural expert systems at CLAES }\end{array}$ & \\
\hline 27 & Rule-based & $\begin{array}{l}\text { translate Greek to Greek Sign } \\
\text { language. }\end{array}$ & \\
\hline 28 & rule based approach. & $\begin{array}{l}\text { well-structured English sentences into } \\
\text { well-structured Arabic sentences }\end{array}$ & \\
\hline 3 & $\begin{array}{l}\text { Grammar parser and example based } \\
\text { machine translation technique }\end{array}$ & $\begin{array}{l}\text { well-structured English sentences into } \\
\text { well-structured Arabic sentences }\end{array}$ & \\
\hline
\end{tabular}




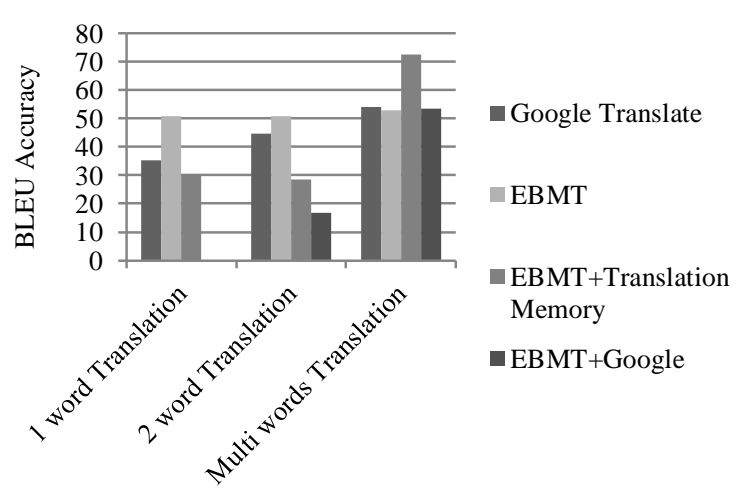

Fig. 3. Comparison between Systems Accuracies for the Second Dataset for Different Input Size in the First Dataset.

Also as shown that when translation from English to Arabic but not in medical domain mot of them used Rule based technique where they analyze the English data in terms of morphology, syntactic and semantic which is not necessary in medical domain.

\section{CONCLUSION AND FuTURE WORK}

A hybrid machine translation system using Example based machine translation technique and Translation memory was introduced in this paper to translate English medical terms to Arabic medical terms in comparison with using Google translate only to translate, Example based machine translation system using matching stage only and finally with a hybrid system using Example based machine translation technique and Google Translate.

The system that used Example based machine translation technique with a Translation memory achieved the highest score in comparison with the other three experiments and this because Translation memory that was used stores the translation of each medical term then when using it to translate the unmatched portions of the input sentence $(\mathrm{Si})$ that were added to the translated text $(\mathrm{St})$ of the closest sentence $(\mathrm{Se})$ from the database in the recombination stage translation of the unmatched portions to the right Arabic medical term will be ensured. For the first dataset the proposed system achieved $77.17 \%$ and for the second dataset $63.85 \%$. Google translate translates some of medical terms according to its English meaning not according to its medical meaning. Also the result from matching stage produces sentences with unmatched words between the input sentence and the closest sentence from the database. Using Google translate also with Example based machine translation translates the some of the unmatched portions according to its English meaning not its medical meaning.

However, using one word translation, two words translation and multi-words translation datasets achieved high score for our system but the multi-words translation dataset achieved the highest accuracy which is $80.93 \%$ for the first dataset and $72.49 \%$ for the second dataset. The reason for that is because the training dataset contains multi-words sentences more the one word sentences and also more than two words sentences.
Adjusting the final result according to the morphology of the Arabic language could make the resulted translation more accurate.

\section{REFERENCES}

[1] Kalyani, Aditi, and Priti S. Sajja., "A review of machine translation systems in India and different translation evaluation methodologies", International Journal of Computer Applications 121, no. 23 ,2015.

[2] Wołk, K. and Marasek, K., "Neural-based machine translation for medical text domain. based on european medicines agency leaflet texts", Procedia Computer Science, 64, pp.2-9, 2015.

[3] Alawneh, Mouiad, Nazlia Omar, T. Sembok, H. Almuhtaseb, and C. Mellish., "Machine translation from English to Arabic", In International Conference on Biomedical Engineering and Technology, 2011.

[4] Arcan, Mihael., "Machine translation of domain-specific expressions within ontologies and documents", PhD diss., 2017.

[5] Neubig, Graham, et al., "Towards high-reliability speech translation in the medical domain", The First Workshop on Natural Language Processing for Medical and Healthcare Fields. 2013.

[6] Dušek O., Hajič J., Hlaváčová J., Novák M., Pecina P., Rosa R., Tamchyna A., Urešová Z., Zeman D., "Machine translation of medical texts in the khresmoi project", In: Ninth Workshop on Statistical Machine Translation, Baltimore, MD, USA Association for Computational Linguistics, p.221-228, 2014 .

[7] Alsohybe, Nabeel T., Neama Abdulaziz Dahan, and Fadl Mutaher BaAlwi, "Machine-translation history and evolution: survey for ArabicEnglish translations", arXiv preprint arXiv:1709.04685,2017.

[8] Sciarra, A.M.P., Batigália, F. and Oliveira, M.A.B.D., "Technological devices improving system of translating languages: what about their usefulness on the applicability in medicine and health sciences?", Brazilian journal of cardiovascular surgery, 30(6), pp.664-667, 2015.

[9] Yepes, Antonio Jimeno, Elise Prieur-Gaston, and Aurélie Névéol, "Combining MEDLINE and publisher data to create parallel corpora for the automatic translation of biomedical text", BMC bioinformatics 1, no. 1: 146, 2013.

[10] Kaur, H. and Laxmi, V., "A survey of machine translation approaches", International Journal of Science, Engineering and Technology Research, 2(3), pp.pp-716, 2013.

[11] Kamran, Amir., "Hybrid Machine Translation”, 2013.

[12] Shaalan, Khaled, Ahmed Rafea, Azza Abdel Moneim, and Hoda Baraka. "Machine translation of English noun phrases into Arabic.". International Journal of Computer Processing of Oriental Languages 17, no. 02, pp: 121-134, 2004.

[13] Gupta, Somya. "A survey of data driven machine Translation." Diss, Indian Institute of ,2010.

[14] Costa-Jussa, M.R., Farrús, M., Mariño, J.B. and Fonollosa, J.A., "Study and comparison of rule-based and statistical Catalan-Spanish machine translation systems", Computing and informatics, 31(2), pp.245-270, 2012.

[15] Dandapat, S., Morrissey, S., Kumar Naskar, S. and Somers, H., "Statistically motivated example-based machine translation using translation memory", 2010.

[16] Seljan, Sanja, and Damir Pavuna. "Translation memory database in the translation process." In Proceedings of the 17th International Conference on Information and Intelligent Systems IIS 2006, pp. 327-332. Croatia, Varaždin: FOI, 2006a, 2006.

[17] Rana Ehab, Eslam Amer, Mahmud Gadallah, "Example-based machine translation: matching stage using internal medicine publications", 7th International Conference on Software and Information Engineering ICSIE, pp. 131-135, 2018.

[18] Névéol, A., Zweigenbaum, P., Max, A., Yvon, F., Ivanishcheva, Y. and Ravaud, P., "Statistical machine translation of systematic reviews into French, training", 15(526), ,p.366K , 2013.

[19] Subalalitha, Aarthi Venkataraman, BasimShahidBaqui, "Statistical machine translation from English to Hindi", International Journal of Pure and Applied Mathematics, vol 118, no. 20, pp. 1649-1655, 2018. 
[20] Renato, A., Castaño, J., Williams, M.D.P.A., Berinsky, H., Gambarte, M.L., Park, H.J., Pérez-Rey, D., Otero, C. and Luna, D.R., "A machine translation approach for medical terms", In HEALTHINF, pp. 369-378, 2018.

[21] Li, J., Kim, S.J., Na, H. and Lee, J.H., "Postech's system description for medical text", Translation Task In Proceedings of the Ninth Workshop on Statistical Machine Translation, pp. 229-232, 2014.

[22] Wołk, K. and Marasek, K.., "Polish-English statistical machine translation of medical texts", In New Research in Multimedia and Internet Systems, Springer,Cham, pp. 169-179, 2015.

[23] Johanna Johnsi Rani G, Gladis D, Joy John Mammen, "Contextsensitive Machine Translation of Medical reports from English to Tamil", International Journal of Pure and Applied Mathematics, vol 119, no. 16, pp. 297-304, 2018.

[24] Mikel Artetxe, Gorka Labaka, Eneko Agirre, and Kyunghyun Cho, "Unsupervised neural machine translation.", In International Conference on Learning Representations (ICLR), 2018.

[25] Amer, E. and Abd-Elfattah, M., "Can wikipedia be a reliable source for translation? testing wikipedia cross lingual coverage of medical domain", IOSR Journal of Computer Engineering (IOSR-JCE), Volume 18, Issue 3, PP 16-22, 2016.

[26] Shaalan, Khaled, Ashraf Hendam, and Ahmed Rafea, "An EnglishArabic bi-directional machine translation tool in the agriculture domain.", In International Conference on Intelligent Information Processing, pp. 281-290. Springer, Berlin, Heidelberg, 2010.
[27] Kouremenos, Dimitrios, Klimis Ntalianis, and Stefanos Kollias, "A novel rule based machine translation scheme from Greek to Greek Sign Language: Production of different types of large corpora and Language Models evaluation", Computer Speech \& Language 51, pp.110-135, 2018.

[28] Al-Taani, Ahmad T., and Zeyad M. Hailat, "A direct English-Arabic machine translation system.", Information Technology Journal 4, no. 3 ,pp: 256-261,2005.

[29] Xuan, H.W., Li, W. and Tang, G.Y., "An advanced review of hybrid machine translation (HMT)", Procedia Engineering, 29, pp.3017-3022, 2012.

[30] Alqudsi, A., Omar, N. and Shaker, K., "Arabic machine translation: a survey, artificial intelligence review”, 42(4), pp.549-572, 2014.

[31] Artetxe, Mikel, Gorka Labaka, and Kepa Sarasola, "Building hybrid machine translation systems by using an EBMT preprocessor to create partial translations", In Proceedings of the 18th Annual Conference of the European Association for Machine Translation, 2015.

[32] Costa-Jussà, M.R. and FMaS, J., "Machine translation in medicine. a quality analysis of statistical machine translation in the medical domain", In Conference on Advanced Research in Scientific Areas (ARSA-2012), 2012.

[33] Wu, Cuijun, Fei Xia, Louise Deleger, and Imre Solti., "Statistical machine translation for biomedical text: are we there yet?", In AMIA Annual Symposium Proceedings, vol. 2011, p. 1290. American Medical Informatics Association, 2011. 